\title{
A New Digital Filter for Unbalance Distorted Current and Voltage Estimation in Power Systems
}

\author{
WAEL M. AL-HASAWI KHALED M. EL-NAGGAR \\ Electrical Engineering Department \\ Collage of Technological Studies \\ Hawali, 32084, P.O. Box 5378, \\ Kuwait
}

\begin{abstract}
This paper presents a novel algorithm for identifying and measuring the symmetrical components of distorted three phase voltage or current waveforms in electrical power systems. The proposed algorithm is capable of estimating the symmetrical components as well as the harmonic contents of a given unbalanced distorted signal. The proposed technique is based on stochastic estimation theorem. The problem is formulated as an estimation problem and presented in state space form. The proposed algorithm used to estimate the positive, negative and zero components of unsymmetrical waveforms as well as the harmonic content of a given distorted signal. Application of the proposed algorithm has been conducted on various test cases. Among which a practical simulated power system has been implemented using EMTP. Various scenarios are carried out to simulate realistic situations of unsymmetrical waveforms. Effects of bad data on the solution accuracy are also studied. The speed of convergence is examined by changing the estimator initial conditions. Results obtained show that the proposed technique can estimate and track the symmetrical components of non-stationary three phase unbalance voltage or current waveforms in noisy environments. Fast accurate solutions are guaranteed regardless of the initial conditions. It is also shown that bad measurements have no effects on the final accuracy of the estimation.
\end{abstract}

Key-Words:- Symmetrical components, identification, estimator, harmonics, protection, unbalanced.

\section{Introduction}

The method of symmetrical components is a very important tool for the analysis of three phase electrical systems. Protection of power system needs accurate identification of symmetrical components of the measured signals. During unbalanced disturbances, symmetrical components change their values significantly. It is thus very important to track symmetrical components on-line. Several methods have, so far, been proposed to calculate the symmetrical components of a voltage or current signals, many of these methods are based on static state estimation, others are based on dynamic state estimation techniques.

Reference [1] presents a review on some of static estimation techniques and proposed the use of recursive least error squares technique. Reference[2] proposed a digital filtering algorithm for estimation of symmetrical components. The algorithm is based on using two digital filters working together for fast estimation. The input three phase unbalanced system is transformed, first, into $\alpha-\beta$ transformation. The least error square estimation technique is then used to identify the magnitude and phase angle of each sequence component. As known, the least error square estimation solution is affected by the presence of bad data. In reference [3] a fast efficient method based on Fast Fourier transform (FFT) is presented. There are basic assumptions embedded in the application of the FFT. These assumptions are (a) the signal is stationary; (b) the sampling frequency is equal to the number of samples multiplied by the fundamental frequency assumed by the algorithm; (c) sampling theorem is satisfied. Misapplication of Fourier transform algorithm would lead to inaccurate results. Reference [4] presents a method based on non-recursive Newton type algorithm. The 
algorithm is not sensitive to power system frequency changes and to harmonic distortion of input signals.

Dynamic state estimation techniques such as Kalman Filtering (KF) and weighted least absolute value dynamic filters are also presented in many references [5], [6] and [7]. The algorithm presented in reference [5] detects the positive and negative sequence components after filtering the zero sequence components. Although this method gives good results, it was not tested in the presence of bad data points. Reference [7] presents an application of a dynamic filter for on-line estimation of symmetrical components. The method detects the sequence components of a pure sine waveforms. Sequence filters are also frequently used in power system protection for identifying voltage or current symmetrical components during abnormal operations. Sequence filters output can be affected by saturation that may happen to the filter coil elements [8]. In reference [8] another on-line method for estimating symmetrical components is presented. The method is based on the use of a set of enhanced phase-locked loop systems. Reference [9] introduced an adaptive linear combiner for symmetrical components estimation. The technique is capable of estimating both the symmetrical components as well as the harmonic contents of measured unsymmetrical distorted signals. The algorithm is based on Kalman filtering technique.

Approaches based on artificial intelligence techniques such as genetic algorithms, fuzzy logic and neural networks are also proposed to detect and identify voltage and current sequence components, [10], [11], [12].Many other techniques are suggested and implemented in the time domain [13], [14].

This paper presents a novel technique based on recursive algorithm which can be used for digital identification of the symmetrical components of the harmonic contaminated voltage or current waveforms in electrical power systems. The algorithm is an optimal dynamic estimator based on stochastic estimation theory which is applicable for estimating and tracking the non-stationary signals. Unlike Kalman Filter which minimizes the error square, The proposed estimator gain matrix is derived in such a way to minimize the absolute error in the estimation process, thus the estimator is named dynamic least absolute estimator (DLAVE). The method allows a very fast determination and isolation of the fundamental and harmonic components and consequently the desired parameters of symmetrical components when a change in the power system occurs. Results obtained show that the proposed technique efficiently estimates the symmetrical components of three phase unbalance voltage or current waveforms under different circumstances.

\section{Mathematical Modeling}

The idea here is to present the relationship between the unsymmetrical waveforms and the symmetrical components in state space form which is suitable for the proposed algorithm. This is done via two steps. In the first step, the distorted signal is decomposed to its fundamental and harmonic contents. In the second step, the fundamental component is resolved to obtain its symmetrical components.

\subsection{Distorted waveform modeling}

Assume that we have a non- sinusoidal waveform given as

$$
i(t)=I_{d c}+\sum_{j=1}^{n} I_{m_{j}} \sin \left(w_{j} t+\theta_{i j}\right)
$$

where

$\mathrm{I}_{\mathrm{dc}} \quad$ is the dc component of the current

$\mathrm{j}$ equals 1 for fundamental and equals $2,3, .$. for harmonics

$\mathrm{n}$ is the maximum order of harmonic considered

$\mathrm{I}_{\mathrm{j}} \quad$ is the $\mathrm{j}^{\text {th }}$ maximum value of the current component

$\theta$ ij is the phase angle of the $\mathrm{j}^{\text {th }}$ current component

Equation 1 can be expanded as:

$$
\begin{aligned}
& i(t)=I_{d c}+I_{m 1} \cos \left(\theta_{i 1}\right) \sin \left(\omega_{1} t\right)+I_{m 1} \sin \left(\theta_{i 1}\right) \cos \left(\omega_{1} t\right) \\
& +I_{m 2} \cos \left(\theta_{i 2}\right) \sin \left(\omega_{2} t\right)+I_{m 2} \sin \left(\theta_{i 2}\right) \cos \left(\omega_{2} t\right)+\ldots \ldots
\end{aligned}
$$

without loosing generality, it can be assumed that the signals are contaminated with harmonics of orders $3^{\text {rd }}, 5^{\text {th }}$ and $7^{\text {th }}$.in addition to the dc offset component, so we can write:

$$
\begin{aligned}
& i(t)=X_{o}+\sin \left(\omega_{1} t\right) X_{i 1}+\cos \left(\omega_{1} t\right) X_{i 2}+\sin \left(\omega_{3} t\right) X_{i 3}+\cos \left(\omega_{3} t\right) X_{i 4} \\
& +\sin \left(\omega_{5} t\right) X_{i 5}+\cos \left(\omega_{5} t\right) X_{i 6}+\sin \left(\omega_{7} t\right) X_{i 7}+\cos \left(\omega_{7} t\right) X_{i 8}
\end{aligned}
$$

where:

$$
\begin{aligned}
& X_{o}=I_{d c} \quad, X_{i k}=I_{m k} \sin \left(\theta_{m k}\right), \quad k=1,3,5,7 \\
& X_{i k}=I_{m k} \sin \left(\theta_{m k}\right), k=2,4,6,8
\end{aligned}
$$

If the current signal is sampled at a pre-selected rate, $\Delta \mathrm{T}$, then $\mathrm{m}$ samples, would be obtained at $\mathrm{t}_{1}$, $\mathrm{t}_{2}, \ldots, \mathrm{t}_{\mathrm{m}}$. In a compact matrix form we can write 


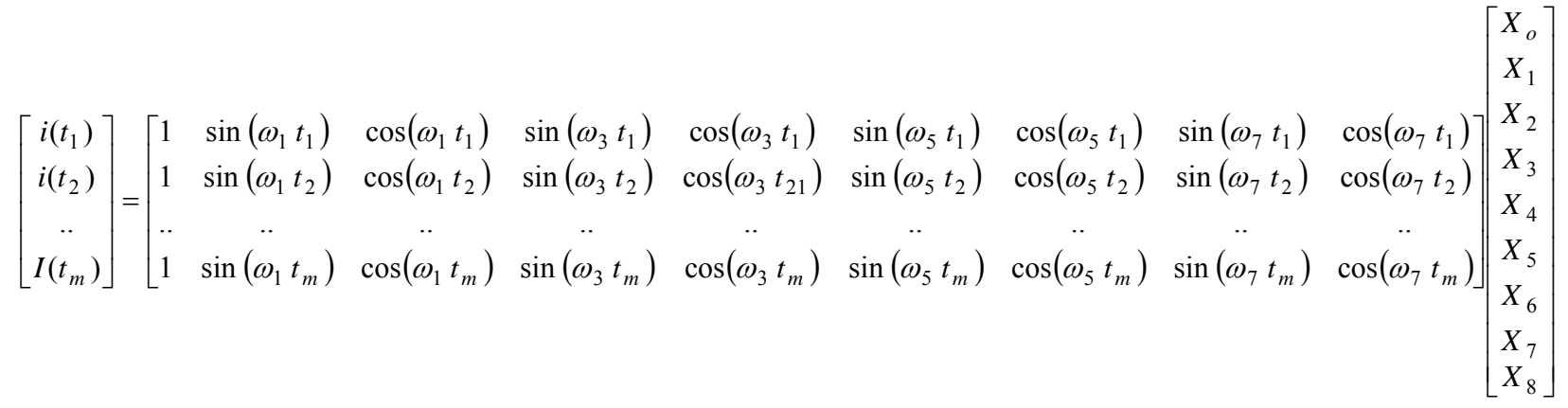

In a compact matrix form, equation 5 can be rewritten in state space form as,

$$
Z(k)=H(k) X(k)+e(k)
$$

where

$\mathrm{Z}(\mathrm{k}) \quad$ is $\mathrm{mx} 1$ measurement vector of the current samples

$\mathrm{H}(\mathrm{k}) \quad$ is $\mathrm{mx} 9$ measurement matrix

$\mathrm{X}(\mathrm{k})$ is $9 \mathrm{x} 1$ state vector to be estimated

$e(k)$ is $m x 1$ measurement error vector, to be minimized. It is assumed to be white sequence with known covariance $\mathrm{R}(\mathrm{k})$.

The state transition equation will be in the form:

$$
X(k+1)=\Phi(k) X(k)+\varpi(k)
$$

where

$\Phi(k) \quad 9 \times 9$ state transition matrix given as a unit diagonal matrix (assuming rotating reference)

$\varpi(k) 9 \times 1$ error vector of the state assumed to be a white (uncorrelated) sequence with known covariance matrix $Q(k)$.

Once the state vector is identified, the RMS values of fundamental, DC and harmonic currents and their phase angles can be calculated as:

$$
I_{k}=\sqrt{X_{k}^{2}+X_{(k+1)}^{2}} \quad, \theta_{i K}=\tan ^{-1}\left(X_{k+1)} / X_{k}\right)
$$

where k equals 1 for fundamental and 3,5,7 for the other harmonics.

Once the fundamental component is separated from the rest of the signal, the symmetrical components can be estimated using the following model.

\subsection{Pure sinusoidal waveform}

Now, Consider a three phase unsymmetrical fundamental voltages or currents given as:

$$
\begin{aligned}
& I_{a}(t)=I_{a m} \sin \left(w t+v_{a}\right) \\
& I_{b}(t)=I_{b m} \sin \left(w t+v_{b}\right) \\
& I_{c}(t)=I_{c m} \sin \left(w t+v_{c}\right)
\end{aligned}
$$

We can write the symmetrical components as:

$$
\begin{aligned}
& I_{a}(t)=I_{0}+I_{1}+I_{2} \\
& I_{b}(t)=I_{0}+a^{2} I_{1}+a I_{2} \\
& I_{c}(t)=I_{0}+a I_{1}+a^{2} I_{2} \\
& \text { Where } \quad a=\exp (j 2 \pi / 3)=1 \angle 120 \\
& I_{a}(t)=I_{0 m} \sin \left(w t+v_{0}\right)+I_{1 m} \sin \left(w t+v_{1}\right)+I_{2 m} \sin \left(w t+v_{2}\right) \\
& I_{b}(t)=I_{0 m} \sin \left(w t+v_{0}\right)+I_{1 m} \sin \left(w t+v_{1}-120\right)+I_{2 m} \\
& \quad \sin \left(w t+v_{2}+120\right) \\
& I_{c}(t)=I_{0 m} \sin \left(w t+v_{0}\right)+I_{1 m} \sin \left(w t+v_{1}+120\right)+I_{2 m} \\
& \quad \sin \left(w t+v_{2}-120\right) \\
& \text { Substitute } \quad \sin (a+b)=\sin (a) \cos (b)+\cos (a) \sin (b) \\
& \cos (a+b)=\cos (a) \cos (b)-\sin (a) \sin (b)
\end{aligned}
$$

We will end up with the following equations(12)

$$
\begin{aligned}
I_{a}(t)= & \boldsymbol{I}_{0 \boldsymbol{m}}\left\{\sin (w t) \cos \left(v_{0}\right)+\cos (w t) \sin \left(v_{0}\right)\right\}+\boldsymbol{I}_{1 m}\left\{\sin (w t) \cos \left(v_{1}\right)+\cos (w t) \sin \left(v_{1}\right)\right\}+\boldsymbol{I}_{2 m}\left\{\sin (w t) \cos \left(v_{2}\right)+\cos (w t) \sin \left(v_{2}\right)\right\} \\
I_{b}(t)= & \boldsymbol{I}_{0 m}\left\{\sin (w t) \cos \left(v_{0}\right)+\cos (w t) \sin \left(v_{0}\right)\right\}+\boldsymbol{I}_{1 m}\left\{\sin (w t) \cos \left(v_{1}\right) \cos (-120)+\right. \\
& \left.\cos (w t) \sin \left(v_{1}\right) \cos (-120)+\cos (w t) \cos \left(v_{1}\right) \sin (-120)-\sin (w t) \sin \left(v_{1}\right) \sin (-120)\right\}+ \\
& \boldsymbol{I}_{2 m}\left\{\sin (w t) \cos \left(v_{2}\right) \cos (120)+\cos (w t) \sin \left(v_{2}\right) \cos (120)+\cos (w t) \cos \left(v_{2}\right) \sin (120)-\sin (w t) \sin \left(v_{2}\right) \sin (120)\right\} \\
I_{c}(t)= & \boldsymbol{I}_{0 m}\left\{\sin (w t) \cos \left(v_{0}\right)+\cos (w t) \sin \left(v_{0}\right)\right\}+\boldsymbol{I}_{1 m}\left\{\sin (w t) \cos \left(v_{1}\right) \cos (120)+\right. \\
& \left.\cos (w t) \sin \left(v_{1}\right) \cos (120)+\cos (w t) \cos \left(v_{1}\right) \sin (120)-\sin (w t) \sin \left(v_{1}\right) \sin (120)\right\}+ \\
& \boldsymbol{I}_{2 m}\left\{\sin (w t) \cos \left(v_{2}\right) \cos (-120)+\cos (w t) \sin \left(v_{2}\right) \cos (-120)+\cos (w t) \cos \left(v_{2}\right) \sin (-120)-\sin (w t) \sin \left(v_{2}\right) \sin (-120)\right\} \quad(12)
\end{aligned}
$$


These equations can be written in a compact matrix form as:

$$
\left[\begin{array}{l}
I_{a}(t) \\
I_{b}(t) \\
I_{c}(t)
\end{array}\right]=\left[\begin{array}{llllll}
h_{11} & h_{12} & h_{13} & h_{14} & h_{15} & h_{16} \\
h_{21} & h_{22} & h_{23} & h_{24} & h_{25} & h_{26} \\
h_{31} & h_{32} & h_{33} & h_{34} & h_{35} & h_{36}
\end{array}\right]\left[\begin{array}{c}
X_{1}(t) \\
X_{2}(t) \\
X_{3}(t) \\
X_{4}(t) \\
X_{5}(t) \\
X_{6}(t)
\end{array}\right]
$$

where

$$
\begin{gathered}
X_{1}(t)=I_{0 m} \cos \left(v_{0}\right) \\
X_{2}(t)=I_{0 m} \sin \left(v_{0}\right) \\
X_{3}(t)=I_{1 m} \cos \left(v_{1}\right) \\
X_{4}(t)=I_{1 m} \sin \left(v_{1}\right) \\
X_{5}(t)=I_{2 m} \cos \left(v_{2}\right) \\
X_{6}(t)=I_{2 m} \sin \left(v_{2}\right)
\end{gathered}
$$

the (h) coefficients are considered as the remaining parts of equations (12). If the three phase currents are sampled at a rate of $\left(f_{s}\right)$ within the specified window size, $\mathrm{m}$ samples would be obtained for each current at $t_{1}, t_{2}, \ldots, t_{m}$, where $t_{1}$ is the initial sampling time. In a state space matrix form we can write this system as in equation (6)

$Z^{\prime}(t)=H^{\prime}(t) X^{\prime}(t)+e^{\prime}(t)$

where:

$Z(t) \quad 3 m \times 1$ vector of current measurements

$H(t) \quad 3 \mathrm{~m} \times 6$ connection matrix

$X^{\prime}(t) \quad 6 \times 1$ state vector to be estimated

$E^{\prime}(t) \quad 3 \mathrm{~m} \times 1$ error vector to be minimized

It is clear that once the state vector $\mathrm{X}(\mathrm{k})$ is estimated, the magnitudes and phase angles of symmetrical components at any step (k) can be calculated as:

$$
\begin{array}{ll}
I_{0}=\sqrt{X_{1}^{2}+X_{2}^{2}}, & v_{0}=\tan ^{-1}\left(X_{2} / X_{1}\right) \\
I_{1}=\sqrt{X_{3}^{2}+X_{4}^{2}}, & v_{1}=\tan ^{-1}\left(X_{4} / X_{3}\right) \\
I_{2}=\sqrt{X_{5}^{2}+X_{6}^{2}}, & v_{2}=\tan ^{-1}\left(X_{6} / X_{5}\right)
\end{array}
$$

\section{Description of the proposed algorithm}

The on-line estimation process of the parameters is performed using the discrete least absolute value filtering algorithm (DLAVF). The complete derivation of the proposed filter equations is beyond the scope of this paper and is given in reference [16]. The dynamic filter works on the discrete state space model described by the measurement equation and the state transition equation in the following form.

$$
\begin{aligned}
& A(k)=H(k) X(k)+e(k) \\
& X(k+1)=\Phi(k) X(k)+\varpi(k)
\end{aligned}
$$

As mentioned before, the measurement error vector $e(k)$ is assumed to be white sequence with known covariance as,

$E\left\{e(k) e(j)^{T}\right\}=\left\{\begin{array}{cc}0 ; & j \neq k \\ R(k) ; & j=k\end{array}\right.$

The initial condition of $X(0)$ is a Gaussian random vector with the following statistics,

$$
\begin{aligned}
& E\{X(0)\}=\bar{X}(0) \\
& E\left\{[X(0)-\bar{X}(0)][X(0)-\bar{X}(0)]^{T}\right\}=\bar{P}(0)
\end{aligned}
$$

where $\bar{P}(0)$ is the initial error covariance matrix of the states, with dimensions $m * m$. The covariance of the error at any step $(k)$ can be obtained by replacing $X(0)$ with $X(k)$ in equation (20). The covariance matrix for $\varpi(k)$ is given as:

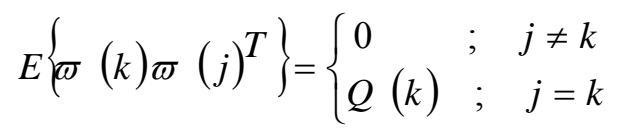

The algorithm starts with an initial estimate for the system parameter vector $\bar{X}(0)$ and its error covariance matrix $(\bar{P}(0))$ at some point $\mathrm{k}=0$. These estimates are denoted as $\bar{X}, \bar{P}$, where $\left(^{-}\right)$means that these are the best estimations at this point, prior to assimilating the measurement at instant $k$. With such initial values, of both parameters and error covariances, filter gain matrix $K(k)$ at this step is calculated as follows,

$$
K(k)=\left[H(k)+R(k) L y^{T} \bar{P}^{-1}(k)\right]^{-1}
$$

assuming that the state vector dimension is $\mathrm{ux} 1$, the vectors $\mathrm{L}$ and $y$ are defined as: $\mathrm{L}$ is $\mathrm{u} x 1$ column vector $(1,1, \Lambda, 1)^{T}$; and $y^{T}$ is $1 x u$ row vector $(1,1)[16]$. Using the filter gains, estimates are updated with measurements $Z(k)$ through equation (23), and error covariances for update estimates are computed from equation (24). 


$$
\begin{aligned}
& \hat{X}(k)=\bar{X}(k)+K(k)[Z(k)-H(k) \bar{X}(k)] \\
& P(k)=[I-K(k) H(k)] P(k)[I-K(k) H(k)]^{T} \\
& +K(k) R(k) K^{T}(K)
\end{aligned}
$$

Finally, the error covariances and the estimates are projected ahead to repeat with $k=2$.

$$
\begin{aligned}
& \bar{P}(k+1)=\Phi(k) P(k) \Phi^{T}(k)+Q(k) \\
& \bar{X}(k+1)=\Phi(k) \hat{X}(k)+R(k)
\end{aligned}
$$

The process is repeated until the last sample is reached. It is assumed that the co-variances and the transition matrices are known. It is also assumed that a good initialization of the filter is obtained using the results of static method such as least squares error or least absolute value. From the test examples, we will show that good initialization is not necessary to satisfy the required accuracy in this application of the filter.

It is very important to mention here that the difference between the proposed discrete least absolute value filter (DLAVF) and Kalman filter (KF) method lies in the gain equation, due to the difference in the nature of the objective function used in deriving the filter equation. In $\mathrm{KF}$, the function is the least squares error, but in DLAVF, the function is the least absolute error.

\section{Testing of Algorithm}

The performance of the proposed method is tested using four different study cases. In the first case a simulated unbalanced three phase currents are generated and analyzed using the proposed algorithm. Effects of bad data on the performance of the estimator are studied.

In the second case, a harmonic contaminated waveforms are analyzed to show the ability of the algorithms for resolving the distorted waveforms and extract the sequence components from it. In the third study case a simulated short circuit currents are generated and analyzed to show the ability of the algorithm to detect the symmetrical components during unsymmetrical short circuits. In the fourth study case, a practical power system, represent the Kuwait extra high voltage network $(275 \mathrm{KV})$, is simulated using EMTP. In this case the testing involves unbalanced waveforms.

\subsection{Case Study 1: Unbalanced load currents} In this case, the algorithm is tested using simulated data based on unbalanced three phase current given in figure 1 .

From $\mathrm{t}=0$ to $\mathrm{t}=0.05$, the currents are given by

$\mathrm{I}_{\mathrm{a}}(\mathrm{t})=100 \sin \left(\mathrm{w}_{\mathrm{o}} \mathrm{t}+30^{\circ}\right), \mathrm{I}_{\mathrm{b}}(\mathrm{t})=50 \sin \left(\mathrm{w}_{\mathrm{o}} \mathrm{t}+300^{\circ}\right)$ and $I_{c}(t)=30 \sin \left(w_{o} t+180^{\circ}\right)$

A sudden change in both magnitude and phase angle is introduced at $\mathrm{t}=0.1 \mathrm{sec}$. in order to test the performance of the algorithm. At $t=0.05$, the new set of currents is given by:

$\mathrm{I}_{\mathrm{a}}(\mathrm{t})=70 \sin \left(\mathrm{w}_{\mathrm{o}} \mathrm{t}+20^{\circ}\right), \quad \mathrm{I}_{\mathrm{b}}(\mathrm{t})=40 \sin \left(\mathrm{w}_{\mathrm{o}} \mathrm{t}-100^{\circ}\right)$ and $I_{c}(t)=10 \sin \left(w_{0} t+70^{\circ}\right)$

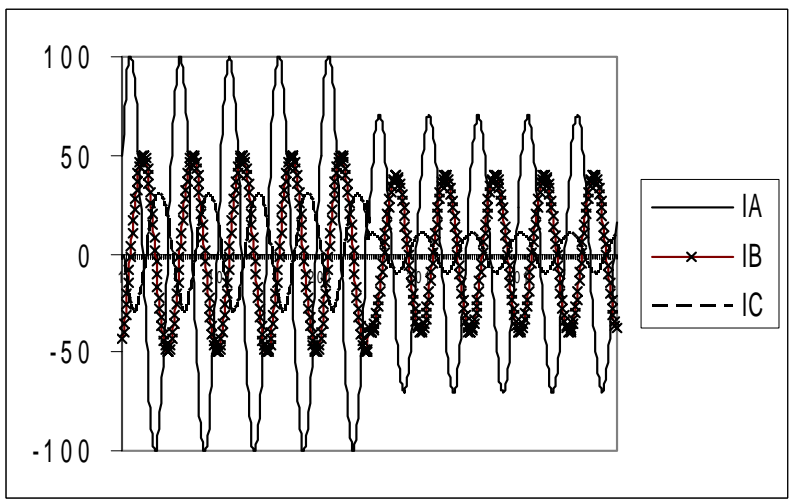

Fig. 1 The generated unsymmetrical waveforms

These currents are sampled at $3000 \mathrm{~Hz}$. The obtained samples are fed to the algorithm. The three magnitudes and phase angles of the zero, positive and negative phase sequence components are shown in figures 2 and 3. Table 1 shows the steady state values of theses estimated magnitudes and angles for both of the periods studied. Figures 2 and 3 show that the algorithm obtained the new sequence components after the sudden change successfully and reached steady state in less than 2 cycles. This means that for non-stationary waveforms, the estimator would track the symmetrical components instantaneously once a change has occurred. The exact solution is thus guaranteed. Examination of table 1 reveals that the results obtained are very accurate. The maximum error is less than $0.5 \%$.

The algorithm is then tested in the presence of bad data. The first period of time ( up-to 0.05 seconds) is used to perform this study. To simulate the bad data situation, the three phase current samples $I_{a}, I_{b}$ and $I_{c}$ are deliberately changed to zeros at randomly selected step, before steady state 
solution is reached. Figure 4 shows the disturbance caused by the bad data points on the estimated symmetrical component magnitudes. It is clear that the final estimation was not affected by bad data points. The only thing is that there is some delay in the time needed to reach the steady state estimation. This can be considered as if the estimator inherently detected the bad data points.

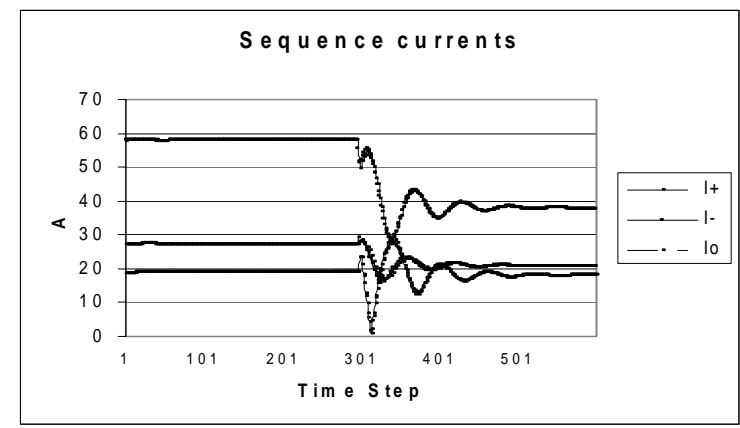

Fig. 2 Symmetrical component magnitudes

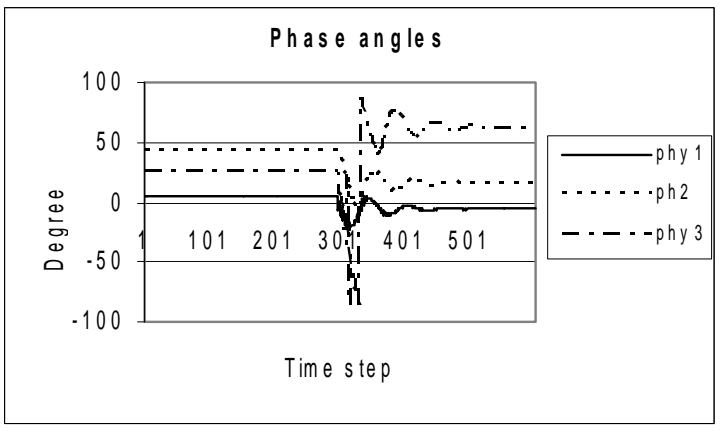

Fig. 3 Symmetrical component phase angles

Table 1 Magnitudes and phase angles of the sequence components

\begin{tabular}{|c|c|c|c|c|}
\hline \multirow{2}{*}{} & \multicolumn{2}{|c|}{$0<\mathrm{t}<0.05$ sec. } & \multicolumn{2}{c|}{$0.05<\mathrm{t}<0.1$ sec. } \\
\cline { 2 - 5 } & Estimated & Exact & Estimated & Exact \\
\hline $\boldsymbol{I}_{\boldsymbol{0}}$ & 27.29226 & 27.2923 & 20.87012 & 20.84866 \\
\hline $\boldsymbol{I}_{\boldsymbol{1}}$ & 57.98314 & 57.9812 & 37.95319 & 37.9362 \\
\hline $\boldsymbol{I}_{\boldsymbol{2}}$ & 18.97255 & 18.9700 & 18.05516 & 18.06 \\
\hline $\boldsymbol{v}_{\boldsymbol{0}}$ & 4.695059 & 4.69280 & -5.52684 & -5.55 \\
\hline $\boldsymbol{v}_{\boldsymbol{1}}$ & 43.29427 & 43.2947 & 15.15746 & 15.26 \\
\hline $\boldsymbol{v}_{\boldsymbol{2}}$ & 24.96427 & 24.9600 & 62.4838 & 62.176 \\
\hline
\end{tabular}

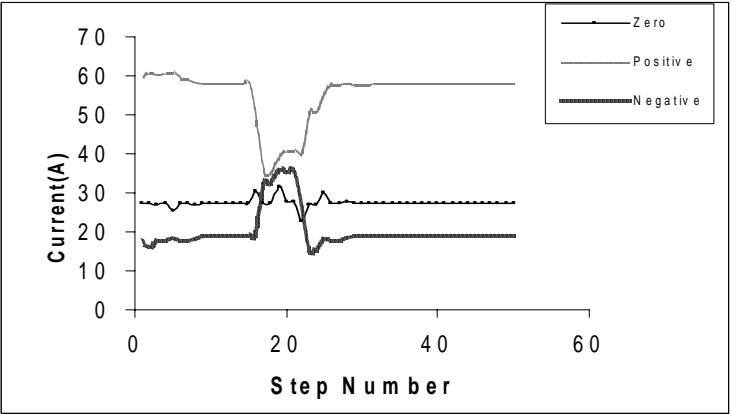

Fig. 4 Bad data effects

\subsection{Case Study 2: Distorted unbalanced currents}

The second study case is aimed toward testing the proposed algorithm in tracking symmetrical components of the current signals contaminated with harmonic distortion, which is a common situation in the actual distribution systems [6], [9]. The data used for this test is taken from reference [6]. Figure 5 shows the considered signal. This signal is assumed to be composed of fundamental and third components. Table 2 shows the estimated fundamental and third harmonic magnitudes and phase angles along with their exact values. It is clear that the fundamental and third harmonic component magnitudes and phase angles are estimated at a very high degree of accuracy. Table 3 shows the final steady state estimate sequence magnitudes and phase angles values. In this case, zero initial conditions were used for the problem states. The convergence to the steady state values is shown in figures $6 a, b, c$. These figures confirm the conclusion reached before. The solution is obtained very fast regardless the initial conditions. The very important thing here is that the filter succeeds in estimating both of the harmonic contents as well as the unsymmetrical components. In other words, the filter can extract the sequence components even from a harmonic contaminated signal.

Table 2 The steady state estimated values of the fundamental \& the harmonic components

\begin{tabular}{|c|c|c|c|c|c|c|c|}
\hline \multicolumn{2}{|c|}{$\begin{array}{c}\text { Fundamental component } \\
\text { Magnitudes }\end{array}$} & \multicolumn{2}{c|}{$\begin{array}{c}\text { Fundamental component } \\
\text { Phase angles (degree) }\end{array}$} & \multicolumn{2}{c|}{$\begin{array}{c}\text { Third harmonic } \\
\text { magnitudes }\end{array}$} & \multicolumn{2}{c|}{$\begin{array}{c}\text { Third harmonic } \\
\text { Phase angles(degree) }\end{array}$} \\
\hline Estimated & Exact & Estimated & Exact & Estimated & Exact & Estimated & Exact \\
\hline 212.128 & 212.132 & 44.98 & 45 & 106.00 & 106.067 & 9.0 & 9.0 \\
\hline 353.547 & 353.55 & 150.06 & 150 & 176.77 & 176.78 & -59.59 & -60 \\
\hline 141.420 & 141.42 & 299.91 & 300 & 70.70 & 70.71 & 120.38 & 120 \\
\hline
\end{tabular}




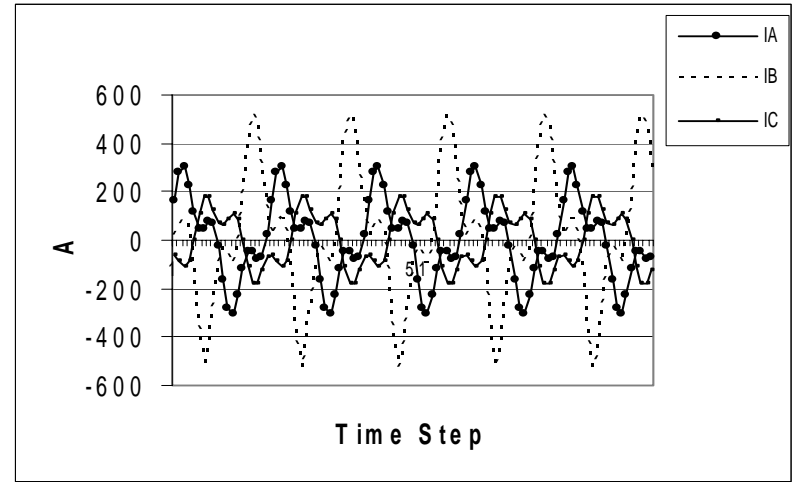

Fig. 5 Three phase distorted unbalanced currents

Table 3 The extracted unsymmetrical components

\begin{tabular}{|c|c|c|}
\hline \multirow{2}{*}{} & \multicolumn{2}{|c|}{ Sequence components } \\
\cline { 2 - 3 } & Estimated & Exact \\
\hline $\boldsymbol{I}_{\boldsymbol{0}}$ & 73.82 & 73.82 \\
\hline $\boldsymbol{I}_{\boldsymbol{1}}$ & 67.91 & 67.91 \\
\hline $\boldsymbol{I}_{\boldsymbol{2}}$ & 230.805 & 230.805 \\
\hline $\boldsymbol{v}_{\boldsymbol{0}}$ & 112.702 & 112.73 \\
\hline $\boldsymbol{v}_{\mathbf{1}}$ & -87.56 & -87.70 \\
\hline $\boldsymbol{v}_{\boldsymbol{2}}$ & 40.45 & 40.50 \\
\hline
\end{tabular}

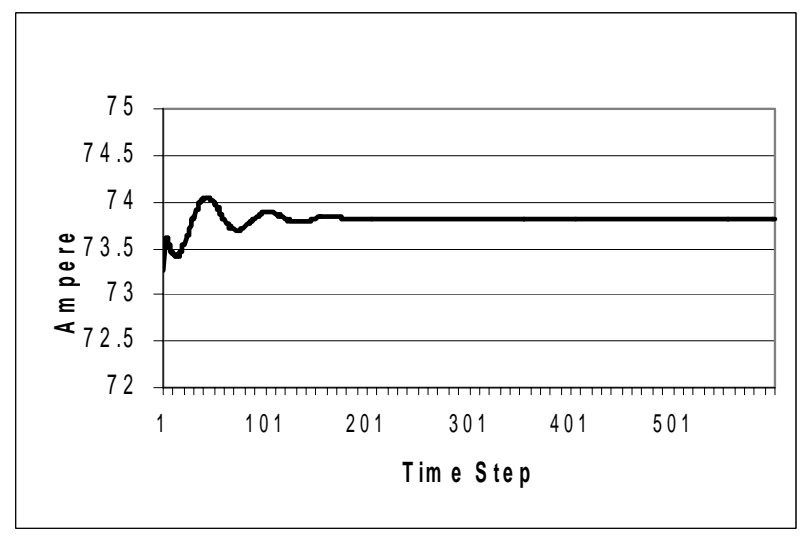

Fig. 6a The zero sequence component

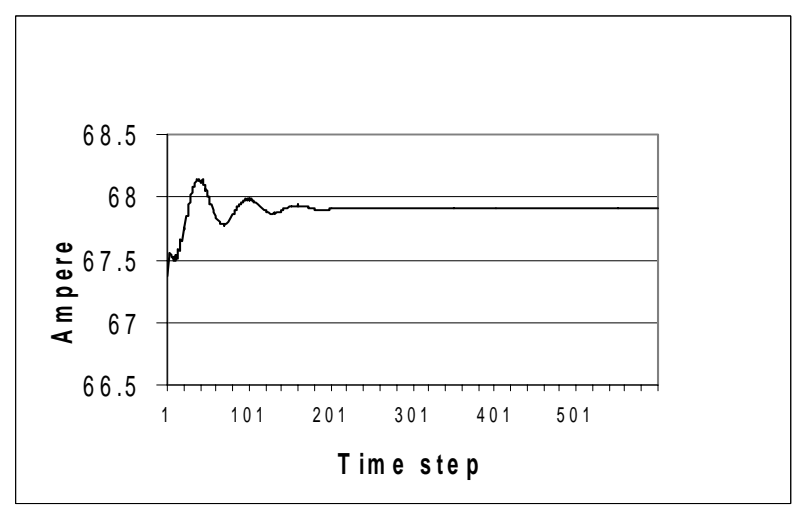

Fig.6b The positive sequence component

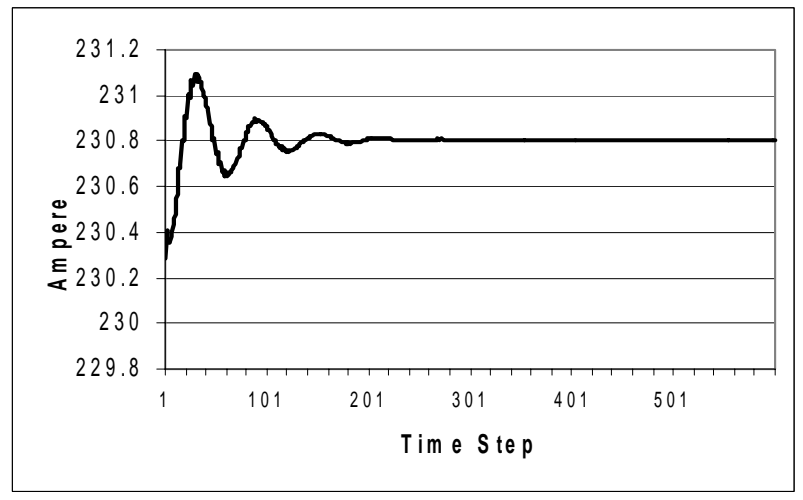

Fig. 6 c The negative sequence components

\subsection{Case study 3: Unsymmetrical short circuit currents}

In this case, a practical simplified power system, taken from the literature $[7,15]$, is used to show the ability of the estimator to track the symmetrical components during short circuits in power system. The system consists of a generator and a step-up transformer which feed a dynamic load, motors, through a transmission line and a step-down transformer. A line to ground fault on phase (A) is assumed to occur at the sending end terminals just after the step-up transformer. Based on the system p.u. data given, a simulation program is used to simulate the three phase unsymmetrical currents measured in the transmission line. The steady state short circuit currents are shown in figure 7.

In reality the measured currents are sampled at a pre-selected rate. A five samples per cycle are used. The power frequency is $50 \mathrm{~Hz}$. Table 4 shows the magnitudes of the symmetrical components and their phase angles as well as the exact values. Examining this table reveals that the results obtained are very accurate compared with the calculated values obtained using the conventional calculations.

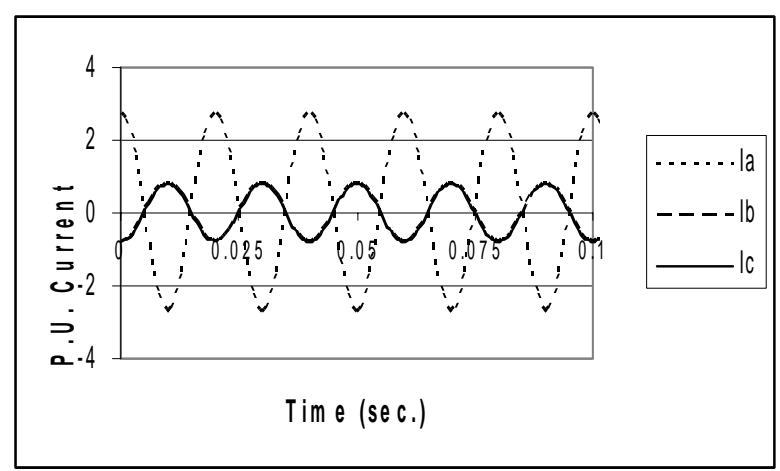

Fig. 7 The steady state short circuit currents 
Table 4 Case study 3, W.S. $=10$ cycles, 5 samples /cycle, zero initial conditions

\begin{tabular}{|c|c|c|}
\hline \multirow{2}{*}{} & \multicolumn{2}{|c|}{ Sequence components } \\
\cline { 2 - 3 } & Estimated & Exact \\
\hline$I_{0}$ & 0.39 & 0.39 \\
\hline$I_{1}$ & 1.18 & 1.18 \\
\hline$I_{2}$ & 1.18 & 1.18 \\
\hline$v_{0}$ & 89.9 & 90 \\
\hline$v_{1}$ & 89.9 & 90 \\
\hline$v_{2}$ & 89.9 & 90 \\
\hline
\end{tabular}

\subsection{Case study 4: Practical Application}

In this case, a practical power system is simulated to demonstrate the ability of the estimator to track the symmetrical components during abnormal conditions in practical power system. The Kuwait EHV network system is simulated using EMTP. The simulated system is shown in figure 8 . The network comprises 15 nodes, 12 double circuit short overhead transmission lines, and 8 underground cable circuits. It is connected in ring system with two main generating stations. The system is used to generate unbalanced current waveforms. The measured unsymmetrical currents at "Alomaria" bus are shown in figure 9. It is clear that the waveforms contains a DC offset. The data window size considered for the analysis is $0.02 \mathrm{sec}$. Table 5 shows the results obtained. It can be noticed that the three components exist. Figure10 shows the solution convergence. Again as in the case of the harmonic contaminated signal, the filter succeeds in estimating the DC offset components as well as the unsymmetrical component of the waveforms. The estimated DC values were 979, 2765 and 1755 Amperes for the phases $a, b$ and $\mathrm{c}$ respectively.

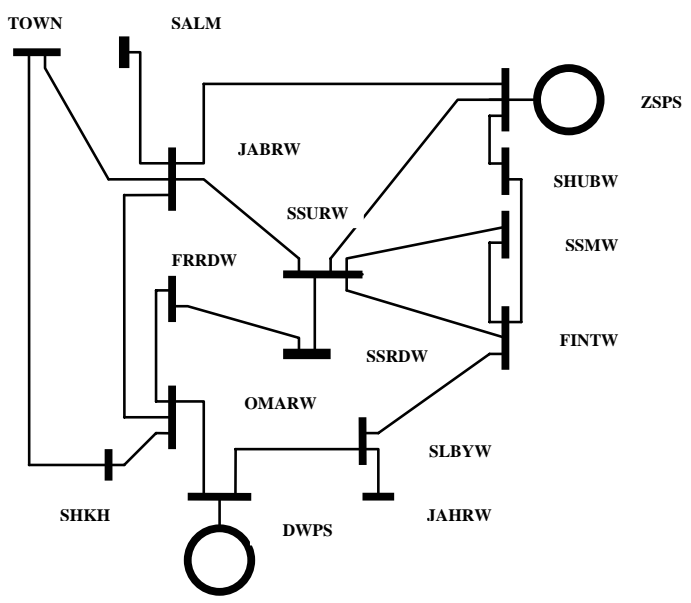

Fig. 8. System under study

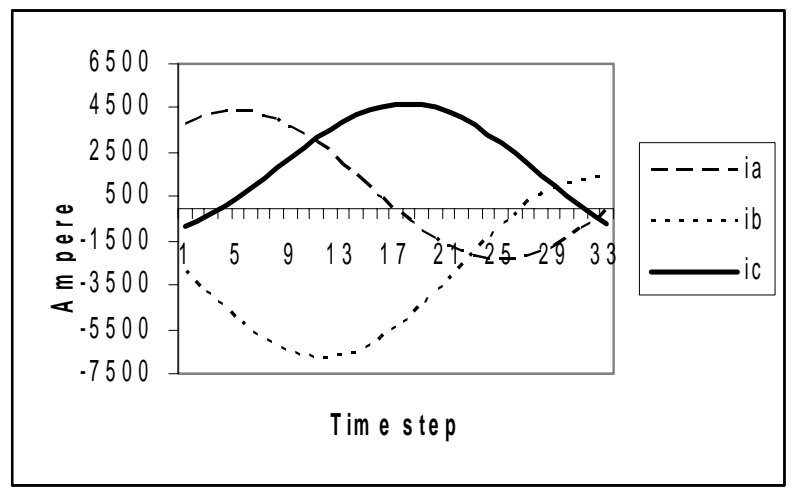

Fig. 9 Unbalanced loading

Table 5 The sequence components of the practical case

\begin{tabular}{|c|c|}
\hline $\boldsymbol{I}_{\boldsymbol{0}}$ & 423.522 \\
\hline $\boldsymbol{I}_{\mathbf{1}}$ & 3437.83 \\
\hline $\boldsymbol{I}_{\boldsymbol{2}}$ & 524.22 \\
\hline $\boldsymbol{v}_{\boldsymbol{0}}$ & $87.02^{\circ}$ \\
\hline $\boldsymbol{v}_{\boldsymbol{1}}$ & $58.90^{\circ}$ \\
\hline $\boldsymbol{v}_{\boldsymbol{2}}$ & $-137.28^{\circ}$ \\
\hline
\end{tabular}

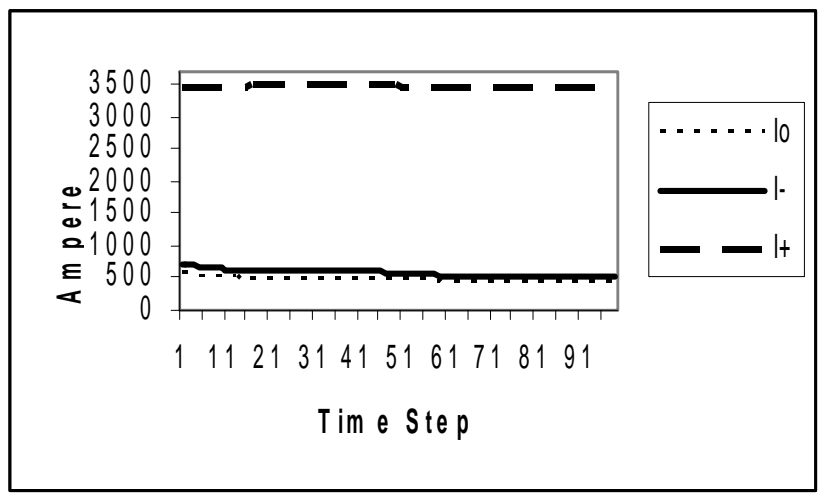

Figure 10 Solution convergence

\section{Conclusions}

In this paper a new dynamic technique is presented for identifying and measuring the symmetrical components of unsymmetrical voltage or current waveforms. The proposed technique is based on stochastic estimation theory. The parameters to be identified are the perpendicular components of the waveform from which both the magnitudes and the phase angles of the symmetrical components are calculated. Simulated data are used to test the algorithm. The algorithm gives very accurate results 
regardless of the initial conditions. This means that if the algorithm is used on-line, it would track the states of the non-stationary waveforms. The estimator has proven that it can give very accurate solutions even with the existence of harmonics, Dc component and bad data points. A practical study cases for determining the symmetrical components of the unsymmetrical currents that flow in practical power systems during unsymmetrical loading and fault conditions, has also been considered. The very accurate results obtained in all cases prove that the proposed method has a very high efficiency that makes it very useful tool in power system protection applications.

\section{REFERENCES:}

[1] S. Sachdev and M. Nagpal, A Recursive Least Error Squares Algorithm for Power System Relaying and Measurements Applications, IEEE Trans., PWDR-6, 1991, pp.1008-1015.

[2] S.A. Soliman, M.A. Mostafa, M.A. EL-Hawary and A. M. AL-Kandari, Two Digital Algorithm for Fast Estimation of Symmetrical Components in a Power System: A Static Estimation Approach, Electric Power Systems Research J., Vol.66, 2003, pp.133-137.

[3] T. Kobos, Fast Estimation of Symmetrical Components in Real Time, IEE Proc.-C, Vol139, No.1, Jan. 1992, pp.27-30.

[4] V.V. Terzija and Markovic, Symmetrical Components Estimation through Non-recursive Newton Type Numerical Algorithm, International Conference on Electrical Power Engineering, PowerTech Budabest 99, IEEE, 1999, pp.248-253.

[5] E. Rosokowski and M. Michalik, Fast Identification of Symmetrical Components by Use of a State Observer, IEE Proc. Generation, Transmission and Distribution, Vol.141, No.6, Nov. 1994, pp.617-622.

[6] S.A. Soliman and M.EL-Hawary, Application of Kalman Filtering for on-Line Estimation of Symmetrical Components for Power System protection, Electric Power Systems Research J., Vol.38, 1997, pp.113-123.

[7] K.M. EL-Naggar, A Fast Method for Identification of Symmetrical Components for power System protection, Electrical Power \& Energy Systems, Vol.23, 2001, 813-817.

[8] M.R. Irvavani and M.Karimi-Ghatemani, Online Estimation of Steady State and instantaneous symmetrical components, IEE Proc. Generation, Transmission and Distribution, Vol.150, No.5, Nov. 2003, pp.616-622.
[9] Mostafa I. Marei, Ehab F. El-Saadany, and Magdy M. A. Salama, "A Processing Unit for Symmetrical Components and Harmonics Estimation Based on a New Adaptive Linear Combiner Structure", IEEE Transactions on Power Delivery, Vol. 19, No. 3, July 2004, pp.1245-1253.

[10] K.M. EL-Naggar and A. R. K. AlOthman, "A Genetic Based Technique For Identification Of Symmetrical Components For Power System Protection", EUROPES04 conference, IASTED, June2004, Rhodes, Greece, pp. 354-360

[11] Alcantara, F.J.; Salmeron, P. , "A new technique for unbalance current and voltage estimation with neural networks", IEEE Transactions on Power Systems, Volume 20, Issue 2, May-2005,.pp.852-858.

[12] P.K. dash, Bmishra, S.K. Banda and M.M. A Salama, Computation of Power Quality Symmetrical Components Using Fuzzy Logic Based Linear Combiners, International Conference on energy Management \& Power Delivery, EMPD '98, IEEE, 1998, pp.17-22.

[13] Lobos, T, "Fast estimation of symmetrical components in real time" IEE Proceedings Generation, Transmission and Distribution], IEE Proceedings C, Volume 139, Issue 1, Jan. 1992, pp. 27 - 30 .

[14] Paap, G.C. , " Symmetrical components in the time domain and their application to power network calculations" IEEE Transactions on power systems, Volume 15, Issue 2, May 2000, pp.522528.

[15] J. Nagrath and D.P. Kothari, Modern Power System Analysis, Second edition, Tata McGrawHill publishing company, 1993.

[16]G.S. Christensen and S.A. Soliman, " Optimal Filtering of Linear Discrete Dynamic Systems Based on Least Absolute Value Approximation", Automatica, Vol. 26, No.2, 1990, pp.389-395. 